\title{
A New Method for Estimation of Temperature in Food Exposed to Abuse Temperature
}

\author{
Hiroshi FuJIKAwA $^{1 *}$ and Yoshihiro KanO ${ }^{2}$ \\ ${ }^{1}$ Tokyo University of Agriculture and Technology, Faculty of Agriculture, Department of Veterinary Medicine, 3-5-8 Saiwai-cho, Fuchu, \\ Tokyo 183-8509, Japan \\ ${ }^{2}$ Toyoseimaiki Corporation, 12 Kuroda, Wakayama-shi, Wakayama 640-8341, Japan
}

Received July 10, 2007; Accepted November 3, 2007

\begin{abstract}
A new method of estimating the temperature in a food (sausage) exposed to dynamic temperatures was developed to predict bacterial growth in the food. First, the temperatures of nodes constructed in the sausage were successfully predicted from the measured temperatures of the surface nodes using the universal heat conduction equation. Second, to apply the method to real food industry contexts, the nodes for measurement were reduced to two standard nodes, namely, the centers of the top and bottom surfaces of the food. Temperature differences between the surface and standard nodes were mostly independent of the exposure temperature. Using the difference data and the temperatures measured at the standard nodes, the temperatures in the food exposed to various patterns of temperature were also successfully predicted. This method is thought to be empirical but practical. A new alert system which predicts the growth of microorganisms contaminating foods might be developed by coupling our method with a microbial growth model.
\end{abstract}

Keywords: Heat transfer, Heat conduction, Temperature estimation, Microbial growth prediction

\section{Introduction}

Food products and their components are often contaminated with microorganisms. Some of these organisms can cause food spoilage and food poisoning outbreaks. The growth of these harmful microorganisms is affected by a number of environmental factors, including the atmosphere (oxygen level), temperature, nutrition level, the hydrogen ion concentration $(\mathrm{pH})$, salt and/or sugar concentrations (water activity, Aw) of the food. However, the values of most of those factors, such as $\mathrm{pH}$ and salt and/or sugar concentrations, are specific to the products and do not change much throughout the process of distribution of the food. The temperature of a food, which can change with its environment, is thought to be the most important factor that can change microbial growth throughout all the processes between the production and consumption of the food.

The temperature of a point in a solid food differs according to the location in the food, and also changes with time. Here, let us consider a case in which a refrigerated sausage on a stainless steel tray is exposed to room temperature for

*To whom correspondence should be addressed.

Email: fujik@cc.tuat.ac.jp a given period. Various types of heat transfer in and around the food are possible. That is, the heat conduction might take place in the food and at the interface between the food and the tray. Heat transfer due to vaporization of water from the food surface and heat convection from the air flowing along the periphery of the food is also possible. Thus, it would be too difficult to estimate the temperature of a point inside the food under such conditions. Among these heat transfers, the heat conduction in the food is thought to predominantly determine the temperature at the point. If the surface temperature of the food is known, the above complex heat transfer problem might be reduced to a simple issue of heat conduction. Namely, the temperatures at the nodes of a space grid constructed in the food could be mathematically estimated from the temperatures of the surface nodes with a universal heat conduction equation. With this concept, we developed a method to estimate the temperature in the sausage product in this study.

Compared with a food exposed to the surrounding air at room temperature, the thermal resistance at the interface of a food exposed to hot water or steam during the production might be smaller; Bellara et al. (1999) considered that the surface temperature of the food interfacing the heating me- 
dium (water or steam) would be equal to that of the medium itself. This made the boundary conditions of the heat transfer for the food much easier.

However, microbial growth in a food is predominantly governed by its temperature, as described above. Many mathematical growth models have been reported so far (Baranyi and Roberts, 1994; Gibson et al., 1987). Recently we developed a new logistic model, which can successfully predict bacterial growth at constant and varying temperatures (Fujikawa et al., 2003 and 2004; Fujikawa and Morozumi, 2005, 2006). Namely, the model could successfully predict the growth of Escherichia coli, Staphylococcus aureus and Salmonella in broth and milk, on the surface of a nutrient agar plate and in a pouched food (mashed potatoes) when exposed to various temperature patterns.

Several investigators have studied thermal inactivation modeling of bacteria coupled to the heat transfer analysis of foods (Bellara et al., 1999; Denys et al., 2005; Mattos et al., 2005; Membré et al., 2006). In all of these studies, food samples, which consisted of cucumber fruit, plastic-pouched food, egg and "agar", were heated in hot water and the temperatures in the foods were estimated mathematically.

To our knowledge, however, few studies have tried to construct a prediction system for heat transfer and microbial growth (Almonacid et al., 2002; Amézquita et al., 2005). Almonacid et al. (2002) simulated Salmonella Enteritidis growth in egg under various temperature conditions, but the temperature predictions in egg were not confirmed with experimental data. Moreover, they thought that the bacteria in the egg would grow exponentially (Almonacid et al., 2002), but actual bacterial growth shows sigmoidal curves. Amézquita et al. (2005) simulated Clostridium perfringens growth during the cooling of cooked boneless ham, but their temperature simulation was two-dimensional.

In this study, we aimed to develop a temperature prediction method using a naked sausage on a stainless steel tray as a model. Sausage products have a possibility of containing microorganisms due to inadequate heat treatment during production (Zanoni et al., 1993). This might allow some of the contaminants inside the products to remain alive after heating. Additionally, a naked sausage, if sterile, could be contaminated with microorganisms on the tray and/or in the surrounding air. If a contaminated sausage is exposed to abuse temperature before serving, the contaminants might increase to a harmful level. Thus, our scenario is that a sausage on a stainless steel tray, which is cooled in a refrigerator, is exposed to abuse temperature for a certain period (10 h).

\section{Materials and Methods}

Food Packaged Bologna sausage with a cylindrical shape (50 mm in diameter) (Ito Ham, Tokyo, Japan) was purchased at a retail store. It was cut into cylinders with a 40-mm height. One slice of sausage was placed at the center of a rectangular stainless steel tray (160 mm in width, 200 $\mathrm{mm}$ in length, $30 \mathrm{~mm}$ in depth, $1.5 \mathrm{~mm}$ in thickness).

Temperature measurement A space grid with cylindrical coordinates of the above sausage was generated. The distances between the nodes in the radial and z-axial (vertical) coordinates of the space grid were both $5 \mathrm{~mm}$. The temperatures of the nodes in the sausage shown in Fig. 1 were measured with type $\mathrm{T}$ thermocouple probes that were inserted in the radial directions and connected to a digital thermometer (NR-600, Keyence, Osaka, Japan) at 30-s intervals for $10 \mathrm{~h}$. The probes were globular with a diameter of about $1 \mathrm{~mm}$. Nodes 1-5, 6-10, and 11-15 were located on the upper surface plane, the middle plane, and the bottom plane, respectively. The distances from nodes $2,3,4$, and 5 to the center (node 1) on the surface plane were 10, 15, 20, $25 \mathrm{~mm}$, respectively. Namely, node 5 was located at the perimeter on the plane. The other nodes, 7-10 and 12-15, were placed in the same manner as nodes 2-5. Node 15 was placed $5 \mathrm{~mm}$ above the bottom plane (Fig. 1), because a peripheral node on the bottom plane was not physically able to be equipped with the thermocouple probe. The temperature of node 16 on the back side of the tray just below the center of the bottom plane (node 11) was also monitored.

The food on the tray was cooled down to about $6{ }^{\circ} \mathrm{C}$ in a refrigerator and stored in a humidified incubator (PR-3G, Tabai Espec, Osaka, Japan) programmed at constant or dynamic temperature for each experiment. The air in the incubator was not mechanically circulated during the experiment.

Temperature prediction (Heat transfer analysis) The temperatures of the nodes of the cylindrical sausage were estimated with the three-dimensional heat conduction law (Eq. 1).

$$
\frac{\partial T}{\partial t}=A\left(\frac{\partial^{2} T}{\partial r^{2}}+\frac{1}{r} \frac{\partial T}{\partial r}+\frac{\partial^{2} T}{\partial z^{2}}\right)
$$

Here, $T$ is the temperature $\left({ }^{\circ} \mathrm{C}\right)$ of a node at time $t(\mathrm{~s}), \mathrm{A}$

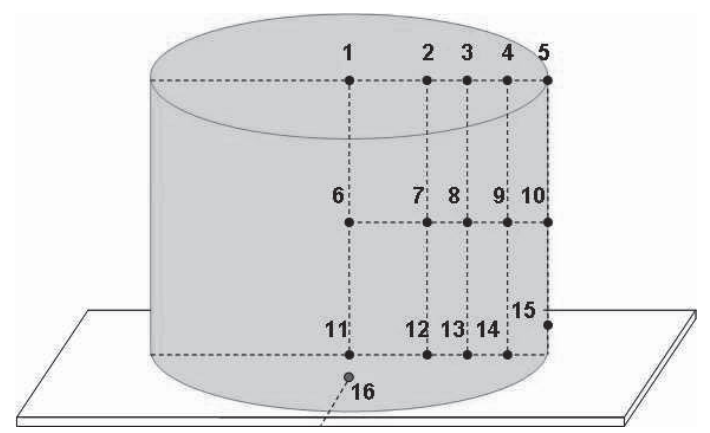

Fig. 1. Nodes constructed in a sausage. The numbers correspond to the locations of the nodes whose temperatures are measured. 
is the thermal diffusivity of the food ( $\mathrm{m} 2 / \mathrm{s}$ ), and $r$ and $z$ are the radial and z-axial coordinates (m), respectively. Equation 1 was numerically solved with the Crank-Nicolson method, which is unconditionally stable (Eq. 2).

$$
\begin{aligned}
& T_{i, j}^{k+1}=\frac{1}{2\left(1+C_{r}+C_{z}\right)}\left[C _ { r } \left\{\left(1+\frac{\Delta r}{2 r_{i}}\right) T_{i+1, j}^{k+1}+\left(1-\frac{\Delta r}{2 r_{i}}\right) T_{i-1, j}^{k+1}\right.\right. \\
& +\left(1+\frac{\Delta r}{2 r_{i}} T_{i+1, j}^{k}+\left(1-\frac{\Delta r}{2 r_{i}}\right) T_{i-1, j}^{k}\right\}+C_{z}\left(T_{i, j+1}^{k+1}+T_{i, j-1}^{k+1}\right. \\
& \left.\left.+T_{i, j+1}^{k}+T_{i, j-1}^{k}\right)+2\left(1-C_{r}-C_{z}\right) T_{i, j}^{k}\right] \\
& \text { where } C_{r}=\frac{A \Delta t}{\Delta r^{2}} C_{z}=\frac{A \Delta t}{\Delta z^{2}}
\end{aligned}
$$

Here, $i$ and $j$ are positive integers presenting the radial and z-axial positions, respectively, of a node in the food. The origin of the space grid was placed at the center of the bottom (node 11 in Fig. 1). $k$ is a positive integer showing the time step. The values of $\Delta t, \Delta r$ and $\Delta z$ were $30 \mathrm{~s}, 5 \mathrm{~mm}$ and $5 \mathrm{~mm}$, respectively. The values of $\Delta r / 2 r i$ in Eq. 2 were set to be one for the nodes along the z-axis and 0.5 for other nodes.

The initial conditions for solving Eq. 2 included with the initial temperatures of all nodes. That is, for nodes whose temperatures were measured (Fig. 1), the measured values were used. Those values were also used for unmeasured nodes symmetric around the centers along the z-axis. The temperatures of other unmeasured nodes were obtained as the averages of the surrounding node temperatures.

The boundary conditions for solving Eq. 2 during the exposure were the temperatures of the surrounding nodes. Namely, similarly to the initial conditions, for the surface nodes where temperatures were measured, the measured values were used. Those values were also used for nodes symmetric around the centers along the $\mathrm{z}$-axis. The temperatures of unmeasured nodes were obtained as the averages of the surrounding surface node temperatures. All calculations were done using a program running on Microsoft Excel.

Method validation The mean square error (MSE) between measured and estimated temperatures of a node during the exposure period was determined to evaluate the accuracy of the estimation. For this validation, four nodes on the middle plane of the food (nodes 6, 7, 8 and 9 in Fig. 1) were chosen.

\section{Results and Discussion}

Estimation of temperatures in the sausage from the surface temperature A refrigerated sausage on a stainless steel tray was exposed to a constant temperature and the temperatures at defined nodes in the food (Fig. 1) were measured. We then estimated the temperatures of the nodes in the food using the measured temperatures of the surface nodes. This would allow us to reduce the problem of temperature estimation of the nodes to simply solving the heat conduction equation (Eq. 1) with the boundary conditions for the temperatures of the surface nodes. The thermal diffusivity, $A$, of the food needed to solve the equation was taken as the value which gave the least MSE between the measured and estimated temperatures of the nodes on the middle plane (nodes 6, 7, 8 and 9 in Fig. 1). This value for $A$ was called the apparent value, $A_{\text {app }}$, in this study. This estimation method (Method I), a conventional one, gave good results at various constant temperatures $\left(10-34{ }^{\circ} \mathrm{C}\right)$. Examples at $22{ }^{\circ} \mathrm{C}$ are shown in Fig. 2. The MSE values for the nodes 6-9 in the figure were $0.16,0.054,0.0031$ and 0.42 , respectively.

The value of $A_{\text {app }}$ increased with the exposure temperature in the temperature range studied (Fig. 3). It was previously
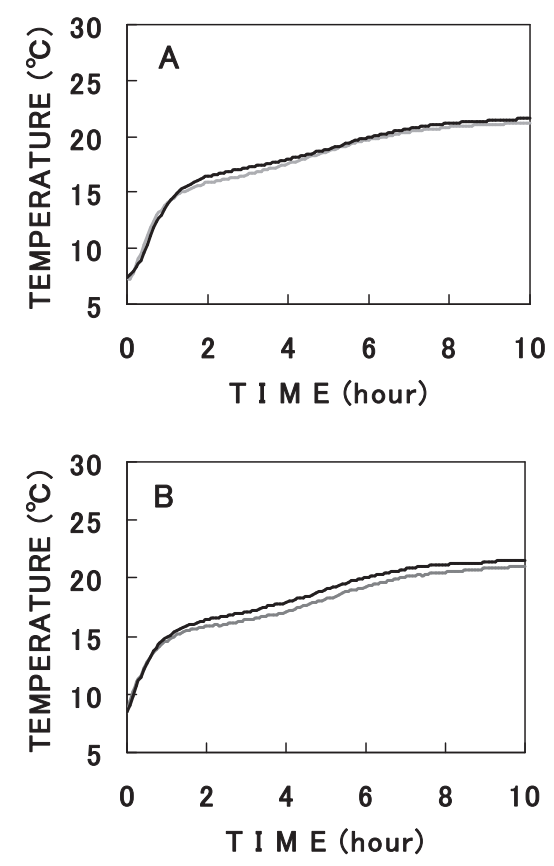

Fig. 2. Temperature estimation for a sausage exposed at $22{ }^{\circ} \mathrm{C}$. The temperatures of the nodes were estimated with Method I. Black and gray lines show estimated and measured temperature patterns, respectively, at nodes 6 (A) and 9 (B).

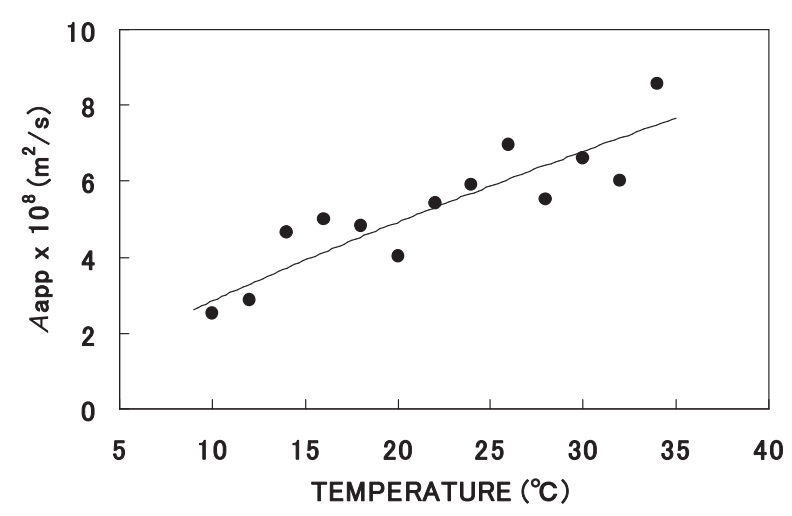

Fig. 3. Temperature dependency of $A_{\text {app }}$. Closed circles are experimental. The curve is the regression line described in the text. 
reported that the value for $A_{\text {app }}$ was smaller than that of $A$ for commercial meat products (for example, approximately $1.3 \times 10^{-7} \mathrm{~m}^{2} / \mathrm{s}$ at $\left.20{ }^{\circ} \mathrm{C}\right)($ Oshita, 1996). The value at a given temperature in this study was expressed using the following exponential equation with the regression coefficient of $\mathrm{R}^{2}=$ 0.813 (Fig. 3).

$$
A_{\text {app }}=0.459 T_{\mathrm{p}}{ }^{0.792}
$$

Here, $T_{\mathrm{p}}$ is the programmed temperature of the incubator. The exponential function here is just for curve fitting.

In a preliminary study, Method I was also found to be successful for pieces of sausage with various heights (2-8 $\mathrm{cm})$. The value of $A_{\text {app }}$ was constant at $2-6 \mathrm{~cm}$ of height and slightly lower at $8 \mathrm{~cm}$ (data not shown). The height of the food was fixed $(4 \mathrm{~cm})$ for subsequent experiments.

The Crank-Nicolson method (Eq. 2) was a good method to solve the differential equation of thermal conduction (Eq. 1). It was robust and gave a precise numerical solution with long time steps. With time steps up to about $60 \mathrm{~s}$, it gave almost the same numerical results. In this study, the time step was therefore fixed at $30 \mathrm{~s}$.

Difference in temperature from the standard nodes Method I was successful, but required measuring the temperature at many surface nodes (11 in the present study) (Fig. 1). This number would be overly large to apply this method for confirming food safety in real food storage and distribution processes. The number of measured nodes needed to decrease. Thus, among the nodes used, two nodes were selected as standard nodes. The standard nodes used were the centers of the top and bottom planes of the food (nodes 1 and 11 in Fig. 1). The temperatures of other peripheral nodes on the top and bottom planes were compared with that of the standard node

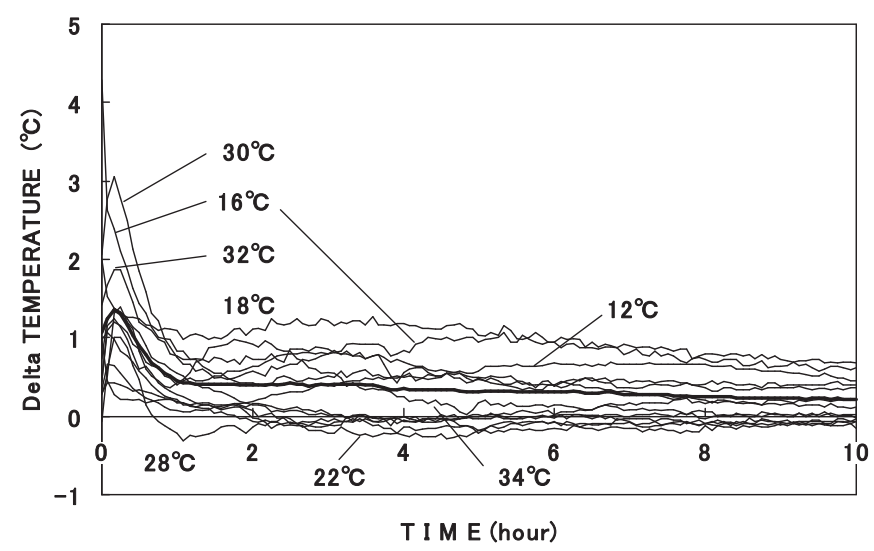

Fig. 4. The temperature differences between node 3 and standard node 1 at various exposure temperatures $\left(10-34{ }^{\circ} \mathrm{C}\right)$. The values of the temperature at node 3 minus that of node 1 are shown as Delta temperature. Some of the curves in the figure are not named because of the crowdedness of the curves. The bold line is the average of all curves. on the corresponding planes. A peripheral node on the middle plane (node 10) was also compared with node 1.

The differences in temperature between the measured nodes and the standard nodes during the exposure were then investigated in the exposure temperature range of 10 to $34{ }^{\circ} \mathrm{C}$. Interestingly, those differences were mostly independent of the exposure temperature. One example at node 3 is shown in Fig. 4. The findings might suggest that the temperature of a surface node could be estimated with the above difference data and the measured temperature of the standard nodes. The average of the temperature difference in the range of 10 to $34{ }^{\circ} \mathrm{C}$ was then obtained for each node (Fig. 5).

Temperature prediction Using the temperature difference data in Fig. 5, we can estimate the temperatures of surface nodes other than the standard nodes using the measured temperatures of the standard nodes at a given temperature. These estimated temperatures of these nodes of the food can be the boundary conditions for solving the heat conduction equation. The value of $A_{\text {app }}$ at a given temperature is estimated from the exposure temperature with Eq. 3. Thus, the temperatures of the nodes can be estimated from these data using Eq. 2. This estimation method is called Method II in
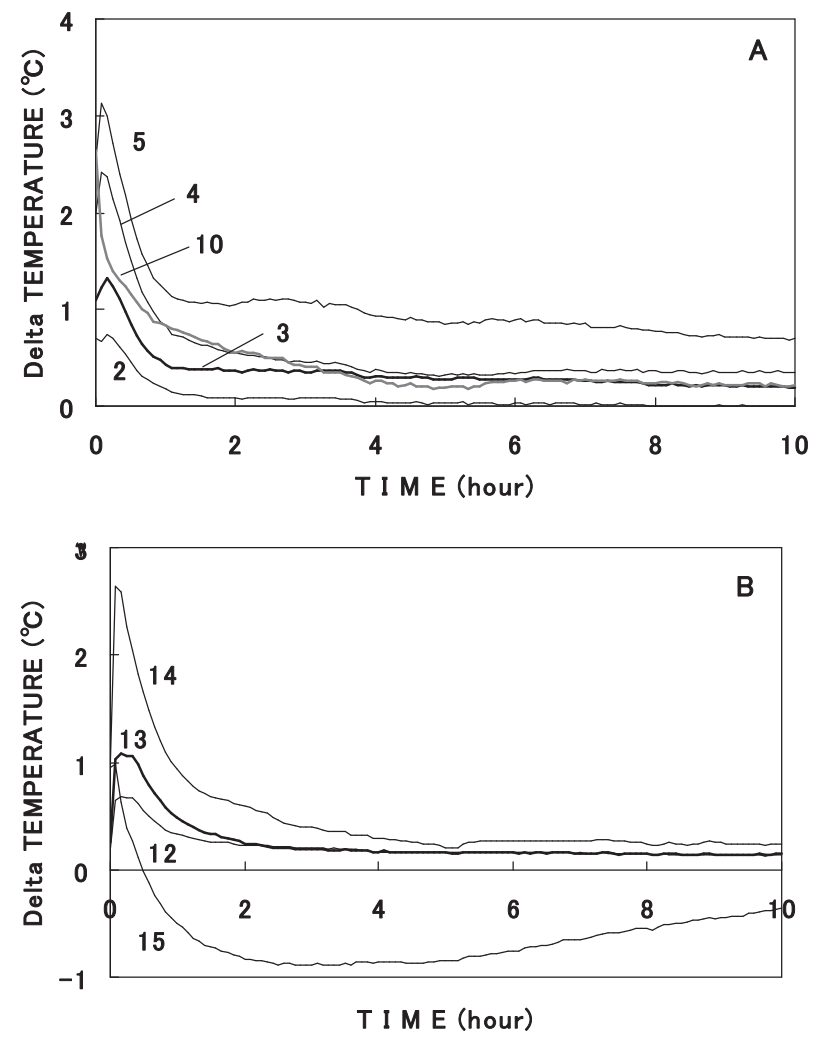

Fig. 5. Temperature differences between the surface and standard nodes. The value of the temperature at nodes $2,3,4,5$ or 10 , minus that of node 1 , is shown in A. In addition, the value of the temperature at nodes $12,13,14$ or 15 , minus that at node 11 , is shown in $\mathrm{B}$. Curves show the averages in the range of $10-34{ }^{\circ} \mathrm{C}$. 
the present study.

Method II was first evaluated for the sausage exposed to constant temperatures. The method successfully predicted temperatures in the sausage at various exposure temperatures. An example, at $27{ }^{\circ} \mathrm{C}$, which was not tested before, is shown in Fig. 6. The MSE values for nodes 6-9 were 0.062, 0.068, 0.075 and 0.082, respectively. Here, $A_{\text {app }}$ at $27{ }^{\circ} \mathrm{C}$ was estimated to be $6.25 \times 10^{-8}\left(\mathrm{~m}^{2} / \mathrm{s}\right)$.

Method II was then evaluated for its prediction at a dynamic temperature. The programmed exposure pattern is shown in Fig. 7T. At the dynamic temperature, Method II also successfully predicted the temperatures of the nodes in the food (Fig. 7A, B). Values of MSE for the nodes 6-9 were $0.26,0.033,0.41$ and 0.13 , respectively. This estimation method was also successful for other dynamic temperature patterns with ups and downs (Fig. 8). Values of MSE for the nodes $6-9$ were $0.59,0.46,0.057$ and 0.20 , respectively. These results suggest that Method II could be a useful tool to predict the temperature in food at various types of exposure temperature patterns. Method II was also successful for solid foods with different shapes. For those foods, an ordinary 3-dimensional space grid could be generated.

In an one-dimensional constant state, the principle would be to apply Newton's law of cooling for the estimation of heat transfer at the boundary between a solid and the sur-
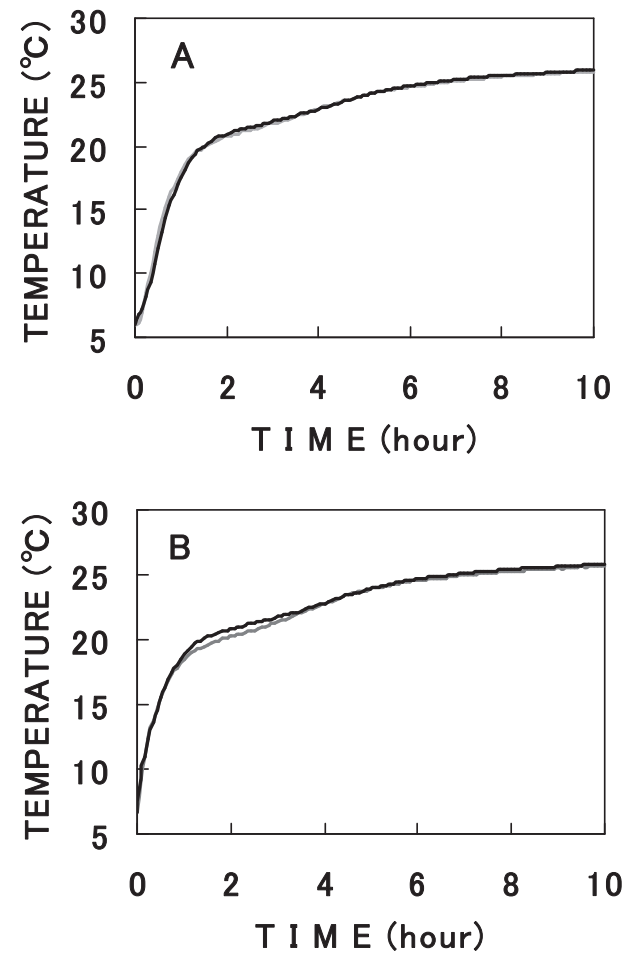

Fig. 6. Temperature prediction in a sausage exposed to $27{ }^{\circ} \mathrm{C}$. The temperatures of the nodes were predicted with Method II. Black and gray lines show predicted and measured temperature patterns, respectively, at nodes 6 (A) and 9 (B). rounding air and Fourier's law of heat transfer to the inside of the solid. In reality, however, it is too difficult to apply these laws for real foods with various shapes, sizes, and thermal characteristics. In this study, we thus introduced the concept of the temperature differences between the nodes in a food from a practical point of view. Our estimation method is thought to be empirical, but practical.

For more effective application of Method II to real food systems, it may be better to replace the measurement at the center of the bottom plane (node 11) with that at node 16 , which is just below node 11 on the back side of the tray (Fig. 1). The reason for this is that the placement of the thermometer probe at node 16 is physically much easier and more realistic. We found that this version was also successful (data not shown).

From the above successful results of Method II, we
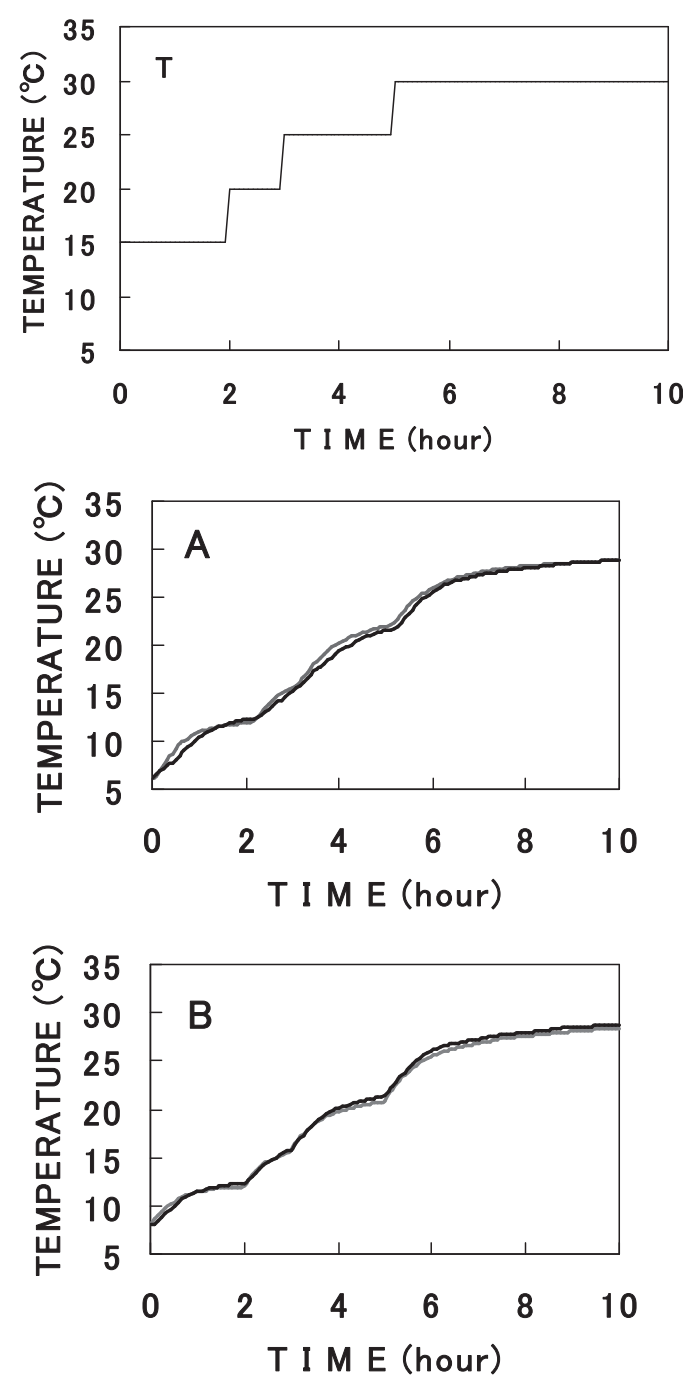

Fig. 7. Temperature prediction in a sausage exposed to a dynamic exposure temperature (I). The programmed temperature history is shown in Fig. 7T. The temperatures of the nodes were predicted with Method II. Black and gray lines show predicted and measured temperature patterns, respectively, at nodes 6 (A) and 9 (B). 

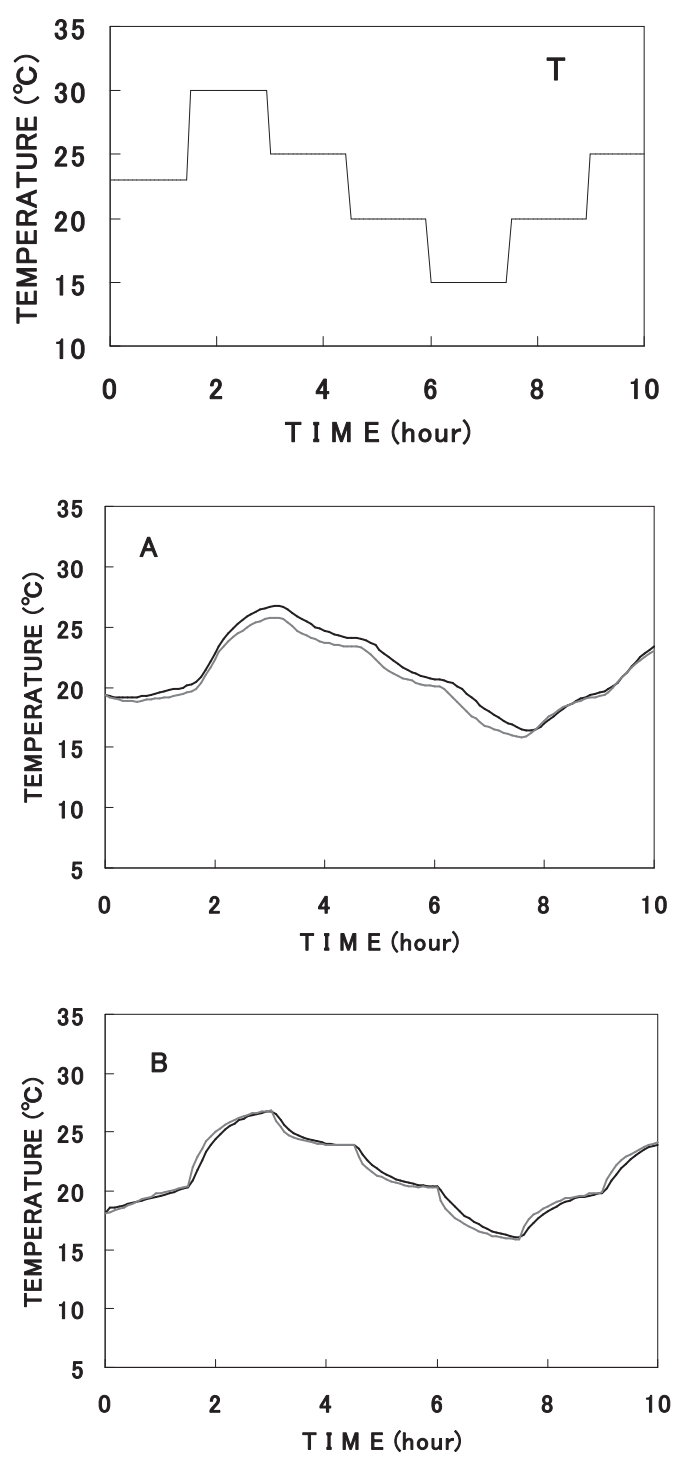

Fig. 8. Temperature prediction in a sausage exposed to a dynamic exposure temperature (II). The programmed temperature history is shown in Fig. 8T. The temperatures of the nodes were predicted with Method II. Black and gray lines show predicted and measured temperature patterns, respectively, at nodes 6 (A) and 9 (B).

preliminarily developed a prediction system consisting of Method II and our microbial growth model described in the Introduction. This might become useful as an alert system to quantitatively predict bacterial growth in a food under dynamic temperature conditions. In the near future, we would like to report on this new system.

Acknowledgements The authors thank Prof. H. Watanabe of the Tokyo University of Marine Science and Technology for reviewing our manuscript, and Prof. Y. Takahashi of the Tokyo University of Agriculture and Technology for supporting this study.

\section{References}

Almonacid, S., Gutierrez, J., Jaques, A., \& Simpson, R. (2002). Salmonella Enteritidis risk assessment: a kinetic analysis. J. Food Sci., 67, 1115-1120.

Amézquita, A., Weller, C. L., Wang, L., Thippareddi, H., Burson, D. E. (2005). Development of an integrated model for heat transfer and dynamic growth of Clostridium perfringens during the cooling of cooked boneless ham. Int. J. Food Microbiol., 101, 123-144.

Baranyi, J., Roberts, T. A. (1994). A dynamic approach to predicting bacterial growth in food. Int. J. Food Microbiol., 23, 277-294.

Bellara, S. R., Fryer, P. J., McFarlane, C. M., Thomas, C. R., Hocking, P. M., Mackey, B. M. (1999). Visualization and modeling of the thermal inactivation of bacteria in a model food. Appl. Environ. Microbiol., 65, 3095-3099.

Denys, S., Pieters, J. G., Dewettinck, K. (2005). Computational fluid dynamics analysis for process impact assessment during thermal pasteurization of intact eggs. J. Food Prot., 68, 366-374.

Fujikawa, H., Kai, A., Morozumi, S. (2003). A new logistic model for bacterial growth. J. Food Hyg. Soc. Jpn, 44, 155-160.

Fujikawa, H., Kai, A., Morozumi, S. (2004). A new logistic model for Escherichia coli at constant and dynamic temperatures. Food Microbiol., 21, 501-509.

Fujikawa, H., Morozumi, S. (2005). Modeling surface growth of Escherichia coli on agar plates. Appl. Environ. Microbiol., 71, 7920-7926.

Fujikawa, H., Morozumi, S. (2006). Modeling Staphylococcus aureus growth and enterotoxin production in milk. Food Microbiol., 23, 260-267.

Gibson, A. M., Bratchell, N., Roberts, T. A. (1987). The effect of sodium chloride and temperature on the rate and extent of growth of Clostridium botulinum type A in pasteurized pork slurry. $J$. Appl. Bacteriol., 62, 479-490.

Mattos, E. R., Fasina, O. O., Reina, L. D., Fleming, H.P., Breidt, F., Jr., Damasceno, G. S., Passos, F. V. (2005). Heat transfer and microbial kinetics modeling to determine the location of microorganisms within cucumber fruit. J. Food Sci., 70, 324-330.

Membré, J.-M. Amézquita, A., Bassett, J., Giavedoni, P., Blackburn, C. W., Gorris, L. G. M. (2006). A probalistic modeling approach in thermal inactivation: estimation of postprocess Bacillus cereus spore prevalence and concentration. J. Food Prot., 69, 118-129.

Oshita, S (1996). Shokuhin no Netubusseichi. In "Shokuhinkanetu no Kagaku,” ed. by S. Shibukawa, Asakura, Tokyo, p. 72 (in Japanese).

Zanoni, B., Garzaroli, C., Anselmi, S., Rondinini, G. (1993). Modeling the growth of Enterococcus faecium in bologna sausage. Appl. Environ. Microbiol., 59, 3411-3417. 